\title{
LA EVIDENCIA EN CENIZAS. DEFINIR Y COMPROBAR EL DELITO DE INCENDIO. VALPARAÍSO, CHILE, 1874-1906*
}

\author{
EVIDENCE IN ASHES. DEFINING AND PROVING ARSON \\ IN VALPARAISO, CHILE, 1874-1906
}

\author{
DIEGO ARANGO LÓPEZ**
}

Resumen: Este artículo estudia simultáneamente la definición penal del delito de incendio y el problema de su comprobación en los tribunales criminales de Valparaíso desde 1868 hasta 1906. Se plantea que incorporar el incendio en el ordenamiento penal chileno supuso dificultades particulares tanto por su definición como por la dificultad que suponía recabar las pruebas. Los incendios, según la lectura que se plantea en esta investigación, son hechos cotidianos que poco a poco, y caso a caso, revelan procesos característicos de la sociedad en la que ocurren. El artículo se propone aportar a una discusión historiográfica poco explorada en Chile y no a la práctica o metodología jurídica actual de acreditación procesal de los incendios. En ese sentido se basa en dos tipos de fuentes. Por una parte, se utilizan los sumarios por incendio recuperados en el archivo judicial criminal de Valparaíso; y, por otra parte, los documentos oficiales correspondientes a la definición penal del delito de incendio con sus soportes teóricos. Finalmente, el artículo permite observar las dificultades específicas que supuso la definición del delito de incendio y, sobre todo, los problemas de la sistematización de procedimientos de demostración judicial.

Palabras clave: Delito de incendio, Valparaíso, Código Penal, seguros contra incendio, siglo XIX.

Aвstract: This article studies the criminal definition of arson and the problem of its verification in the criminal courts of Valparaíso from 1868 to 1906. I argue that incorporating arson into the Chilean criminal law entailed difficulties both because of its definition and the difficulties for collecting and analyzing evidence. Fires, according this research are daily events that progressively and case by case bases, reveal characteristic processes of the society in which they occur. This article intends to contribute to a scarce historiographical discussion in Chile and not to the current methodology on

\footnotetext{
* Este trabajo es resultado del proyecto Fondecyt Postdoctorado N³180471, "La ciudad inflamable. Arquitectura, urbanismo e infraestructura contra-incendios en Valparaíso. 1843-1931"; y del proyecto FIIC 2019-02-09 de la Academia de Humanismo Cristiano: "Incendiarios en Valparaíso. Historia del delito de incendio. 1861-1906".

** Doctor en Estudios Urbanos. Académico de la Universidad Academia de Humanismo Cristiano, Santiago, Chile. Correo electrónico: diego.arango@uacademia.cl. Orcid: https://orcid.org/0000-0001$5831-6073$
} 
judicial verification of arson, therefore it is based on two types of sources. On the one hand, the judicial summaries for criminal arson recovered in the criminal judicial file of Valparaíso. And on the other, the official documents corresponding to the criminal definition of arson are used with their theoretical supports. Finally, the article allows us to observe the specific difficulties that the definition of arson entailed and, above all, the problems of the systematization of judicial proving procedures.

Keywords: Arson, Valparaíso, Penal Code, Fire insurance, 19th Century.

Recibido: 07.11.20. Aceptado: 14.07.2021.

\section{INTRODUCCIÓN}

$A_{\text {tes en la ciudad de Valparaíso por distintas causas (Arango, 2020). Una }}^{\text {FINES de }}$ de ellas fue el incendio criminal. Los incendios, rápidamente, empezaron a llegar a los tribunales del crimen de Valparaíso. Pero las razones para presentar una querella por esta causa eran diversas, entre ellas estaban desde la cobranza fraudulenta de un seguro contra incendio hasta la venganza personal ante una injusticia percibida. Por ejemplo, en 1898, Estanislao Fraga se querelló contra su exmayordomo basándose simplemente en sospechas y rumores repetidos en una cantina local, pues escuchó decir que era este quien había prendido fuego a su casa en un arrebato de resentimiento y alcohol (Incendio en casa de Don Estanislao Fraga "Población Rocuant", 1898). En este caso, como en la mayoría de los procesos de incendio criminal, la demostración judicial suponía un verdadero desafío, pues, de las evidencias físicas, generalmente, no quedaban más que cenizas mojadas. Efectivamente, durante el siglo XIX, la dificultad para juzgar un delito de incendio en Valparaíso repercutió directamente en el desarrollo de una discusión teórica sobre la definición del incendio mismo, pero también sobre el ejercicio de la justicia en terreno. Además, la dificultad para encontrar pruebas contundentes que demostraran la participación de individuos en el delito condujo a los tribunales de Valparaíso a desarrollar mecanismos diversos para recabar y contrastar evidencia testimonial y documental, de esta manera, la judicialización de incendios y el proceso probatorio se convirtieron en una riquísima fuente de relatos sobre las relaciones sociales y culturales de la ciudad.

Ahora bien, el delito de incendio se incorporó en el Código Penal desde su promulgación en 1874, así Chile fue uno de los primeros países de la región latinoamericana y del Caribe, solo después de Haití (1835) y Bolivia 
(1834), en codificarlo. Sin embargo, en la historiografía local, es poco lo que se sabe acerca del proceso de tipificación de este delito durante el siglo XIX. Mucho menos es lo que se conoce acerca de la práctica de justicia en casos de incendio en Valparaíso. En cuanto a la práctica, específicamente, el artículo se interesa por los procedimientos para la demostración en terreno, haciendo énfasis en las dificultades para la inspección de propiedades incendiadas, el manejo de evidencias y la creación de narrativas argumentativas en casos de incendio. Cabe señalar que este artículo no busca aportar a las ciencias jurídicas para desarrollar metodologías de comprobación y acreditación procesal de los incendios. Se trata de una investigación de corte historiográfico que pretende analizar algunos elementos fundamentales del proceso histórico de incorporación del delito de incendio al orden jurídico penal chileno desde finales del siglo XIX hasta principios del XX.

Ahora bien, algunos autores han abordado someramente aspectos de la historia del delito de incendio en Chile, resaltando, especialmente, el problema de la codificación del incendio en el Código Penal chileno (Aliaga et al., 1974; Espinoza, 1952; Valdovinos, 1938). Sin embargo, aunque se han realizado importantes aportes a la práctica jurídica actual para definir criterios de imputación de responsabilidad en casos de incendio y generar técnicas y medios de prueba en procedimientos y peritajes, no existe una investigación historiográfica que permita comprender las dificultades específicas de la práctica judicial en caso de incendio en el periodo determinado. Respecto de Valparaíso en particular, aunque el archivo judicial contiene múltiples expedientes de incendios criminales, la historiografía, por el momento, no ha estudiado en detalle los procedimientos de los tribunales. Temas cercanos, como el fraude al seguro mediante el incendio, han sido observados parcialmente desde la historia del derecho comercial y económica, y desde la historia de los seguros contra incendio (Llorca, 2010, 2011).

Sin embargo, la inclusión del incendio como delito en el Código Penal tuvo repercusiones importantes en la construcción de una forma de pensar el incendio, y en la práctica general de la justicia en terreno. Agentes de policía, expertos, peritos, jueces, incendiarios, aseguradores y víctimas se veían enfrentados una y otra vez a la necesidad de inspeccionar lugares siniestrados, demostrar inocencia, grados de culpabilidad o responsabilidad en la comisión de un delito de incendio.

Esta investigación se basó en dos tipos de fuentes. Por una parte, para comprender el proceso de construcción del concepto de delito de incendio se utilizaron documentos oficiales como el Código Penal, las actas de la 
comisión redactora del código penal, y la discusión parlamentaria correspondiente. Por otra parte, el análisis de la práctica judicial misma se hizo a través de una selección de expedientes judiciales recopilados en el archivo judicial criminal de Valparaíso. Además, el análisis de casos se complementa con documentos como pólizas de seguros contra incendio, documentación de las aseguradoras, y diferentes informes y comentarios generados por los bomberos de Valparaíso en sus libros de actividad.

\section{DEFINIR PARA PENALIZAR}

La práctica de la justicia en Chile funcionaba, desde los tiempos de la administración borbónica, con base en una lógica jurisdiccional de poder en el terreno local que se había corroborado con ajustes en el Reglamento de Administración de Justicia de 1824 (Brangier, 2014, p. 40). En términos generales, esto significaba que los jueces actuaban en conocimiento de las costumbres, expectativas, poderes y legitimidades locales, pero con frecuencia desconocían el texto exacto de la ley que debían aplicar. Específicamente, en términos de comprobación del incendio criminal, el reglamento de 1824 no decía nada.

De hecho, hasta la década de 1870, para dictar un veredicto sobre un incendio, los jueces criminales de Valparaíso no acudían a códigos procesales. Sin embargo, un juez podía valerse de otras reglamentaciones locales o nacionales, como los artículos 599 a 602 del Código Civil, que establecían ciertas normas para la construcción (Código Civil, 1856), y que por lo tanto permitían constatar si una casa se ajustaba a normas que buscaban garantizar la seguridad de las edificaciones; podía acudir también al reglamento de policía urbana de la ciudad (Intendencia de Valparaíso, 1844) que sancionaba prácticas calificadas como peligrosas; o basarse en la ordenanza de la Intendencia de Valparaíso de enero de 1859 que regulaba aspectos de la construcción ignífuga (Ordenanza contra incendios dictada por la Municipalidad de Valparaíso, 1859). Pero, en términos estrictamente penales, el juez no contaba con herramientas específicas para determinar la responsabilidad de un individuo en un incendio. De hecho, ninguna de estas normas y reglamentaciones refería directamente al acto de incendiar una propiedad. Para resolver querellas y establecer penas por incendio criminal, no había hasta entonces ninguna fórmula predefinida en el sistema de derecho de la República de Chile. 
Efectivamente, definir el concepto de incendio suscitaba diferencias de fundamentación ante las cuales los nacientes códigos penales de Europa y América no parecían estar de acuerdo. Esto no significaba que el incendio fuera un problema nuevo, de hecho, desde 1838 existían en Valparaíso mecanismos de inspección y control de edificaciones para evitar incendios (Intendencia de Valparaíso, 1838), pero en términos estrictamente penales, tan solo a partir de 1875 la justicia chilena contaría con una codificación del delito de incendio. Este cambio respondía, ciertamente, a la necesidad de sistematizar la justicia que suponía la consolidación de un Estado-Nación moderno, pero también, a la creciente importancia de la protección de la propiedad que requería la consolidación del nuevo orden comercial del capitalismo global. Por lo tanto, aunque el incendio podía ser interpretado como un crimen contra la seguridad pública, como lo hacía el Código alemán de 1871, la mayoría de códigos del siglo XIX que lo consideraban Francia 1810; Bolivia 1834; Haití 1834; España 1848; Austria 1852; Bélgica 1867; República Dominicana 1884- lo catalogaban como un delito contra la propiedad (Aliaga et al., 1974). Asimismo, no se puede afirmar que el delito de incendio y la protección penal de la propiedad fuera una problemática particularmente chilena, ni específicamente de Valparaíso, pero sí es claro que en esta ciudad el problema de los incendios se presentaba tempranamente y con mucha frecuencia (Arango, 2019).

Algunos casos, como el del incendio del despacho de Eugenio Barruel, ocurrido en diciembre de 1868, ya mostraban las dificultades que tenían los tribunales para determinar la culpabilidad y asimismo dejaban en evidencia las arbitrariedades en que, por falta de pruebas contundentes, podían incurrir incluso los jueces más experimentados y renombrados, como Eulogio Altamirano. En este caso, tras una condena por incendio en primera instancia, David Grimwood, el abogado defensor, señaló los vicios del procedimiento probatorio de la siguiente manera:

Cuanto más examino este cargo menos fundamento le hallo para deducirlo como tal. El que dos individuos habiten una pieza donde ha tenido lugar un incendio[,] el que salga[n] de ella y tornen en seguida volviendo a salir después, no es antecedente alguno para imputar el incendio a aquellos mismos individuos. En los mil procesos que el juzgado debe haber instruido para averiguar el origen de otros tantos incendios ocurridos en este puerto, tal vez no halla uno solo en que la casa [,] tienda o despacho incendiado no hayan sido evitados por alguna persona, $y$ en que el siniestro no haya tenido lugar en un momento de ausencia de 
los habitantes que se ignora hasta este momento que se haya hecho recuerdo pena de incendiario sobre una persona cualquiera nada mas que porque habitaba la posesión incendiada i por que salió de ella, volvió y volvió a salir. (Grimwood, 1868)

En esta ocasión el abogado Grimwood refutaba, uno por uno, los cargos imputados a sus defendidos argumentando que ninguna de las conductas demostradas en los testimonios y evidencias recabados era prueba irrefutable de que hubieran iniciado el incendio. Aceptaba, por ejemplo, que los comportamientos de sus clientes eran inmorales, pues en lugar de cuidar el despacho como se los había solicitado su padre, los jóvenes se habían ido en busca de prostitución; inmorales sí, incendiarios no. Grimwood reconocía también que había algo de estupidez en la conducta de Ernesto Barruel y Luis Dusseaux en el juzgado, ya que no habían dicho la verdad en un principio, y hasta admitía que podía haberse incurrido en otros delitos como el cohecho, pues habían intentado sobornar a un agente del cuerpo de serenos que estaba operando como agente fiscal para recabar testimonios, pero señalaba que esto de ninguna manera comprobaba su participación en el incendio. Finalmente, la Corte de Apelaciones no tenía más alternativa que absolverlos y sobreseer el caso. Más allá de las particularidades específicas de un caso, este expediente demuestra que, con la evidencia en cenizas, los jueces de Valparaíso no contaban con elementos suficientes para determinar la culpabilidad de un presunto incendiario, ni siquiera podían determinar las causas del incendio con total certeza. Y, como bien lo señalaba Grimwood, de acuerdo con la legislación vigente el crimen de incendio no podía ser procesado estableciendo la presunción de culpabilidad de los acusados.

Sin embargo, la discusión sobre el delito de incendio llegaría rápidamente a la esfera penal nacional, en parte, gracias a jueces como el del caso Barruel, Eulogio Altamirano, quien también era autor del Código Penal. Para empezar, en la sesión preparatoria para su redacción, sus autores resaltaron la importancia de utilizar códigos europeos y convinieron en tomar como base el Código Penal español (Comisión redactora del código penal, 1873, pp. $189-190)^{1}$. De este modo, siete artículos del Código español, en cuanto

${ }^{1}$ A las sesiones de redacción del código, en la parte sobre incendios, acudieron el senador Alejandro Reyes, el ministro del interior Eulogio Altamirano, los señores Manuel Renjifo, José Antonio Gandarillas y Adolfo Ibáñez. En términos generales, el procedimiento consistía en retomar y discutir los artículos de los códigos belga de 1867 o español de 1848 para generar el proyecto de Código. Posteriormente, el documento sería sometido a discusión y aprobación del Congreso Nacional. 
a incendios se refiere, serían retomados casi idénticamente en el Código Penal chileno. Sin embargo, la importación y aplicación de un modelo extranjero sería compleja y, como se podría constatar en terreno, no bastaría con copiar los distintos artículos para tener un instrumento conveniente.

\section{ATENDIDAS LAS CIRCUNSTANCIAS}

La simple asignación de penas para casos de incendio resaltaba debates de fondo sobre la definición del delito, e incluso, sobre la naturaleza misma del Código Penal. Para empezar, la asignación de la pena de muerte suscitaba el siguiente debate entre los comisionados: “(...) pidió el señor Reyes que se impusiera en todo caso la pena de muerte, i el señor Gandarillas solo cuando resulten homicidios a consecuencia del incendio; (...)" (Comisión redactora del código penal, 1873, pp. 189-190). Este problema se presentaba, en primer lugar, porque el incendio podía definirse como un resultado, pero no siempre como un acto. Para Ibáñez, por ejemplo, un fallecimiento como efecto del incendio era razón suficiente para sentenciar a muerte al responsable, aunque este no fuera el móvil del crimen (Comisión redactora del código penal, 1873, p. 190). En cambio, en la interpretación de Gandarillas, la pena de muerte solo debía corresponder en caso de homicidio deliberado mediante incendio. Pero ¿cómo determinar si el fallecimiento de una persona en medio de una situación de incendio era o no un homicidio?

Efectivamente, en este delito, tan común pero tan diverso, la muerte podía presentarse de maneras muy disímiles. ¿Era una muerte por asfixia un homicidio mediante incendio? $\mathrm{O} i$ es homicidio si alguien salta de un balcón por miedo a las llamas? Los comisionados comprendían la dificultad ante la cual se enfrentaban, sin embargo, parecían reconocer que no tenían las herramientas necesarias para resolver el problema. Asimismo, comprendían que no podrían ofrecerles a los jueces un instrumento para inspeccionar causas en este tipo de casos. Por lo tanto, Gandarillas propuso una solución simple y solicitó que se agregara la frase "atendidas las circunstancias" en el artículo, para que se entendiera que los jueces no tenían discrecionalidad total pero sí debían realizar procedimientos de comprobación para observar las condiciones generales en las que se había presentado el incendio (Comisión redactora del código penal, 1873, pp. 190-191).

Pero esta solicitud daba origen a un problema de fondo, pues, más allá de especificar la pena por un delito determinado, "atender las circunstancias" significaba establecer un trabajo de comprobación en terreno que re- 
quería técnicas que, por el momento, no estaban definidas. Ahora bien, la reflexión sobre la comprobación del incendio también condujo a la comisión a señalar en el libro primero del Código que siempre que se indicara que alguna pena quedaba a elección del tribunal, este último debía tener en consideración las circunstancias del delito. La dificultad, sin embargo, consistía en definir cómo tener en cuenta de manera justa y sistemática las circunstancias de un incendio. ¿Cómo valorar, por ejemplo, factores meteorológicos como la humedad o el viento que incidían en la inflamabilidad de los objetos y en la comunicabilidad del fuego?, ¿cómo considerar factores urbanos como la combustibilidad de los materiales de construcción, la cercanía de las casas, la accesibilidad de material de bomberos o el acceso a infraestructuras hidráulicas?, ¿cómo incorporar factores económicos como las pólizas de seguros o el valor de la propiedad incendiada?, o ¿cómo sopesar los análisis de agentes de seguros o de bomberos? Estos elementos eran discutidos con frecuencia, pero de manera muy diversa en los distintos casos que llegaban al juzgado. En el caso del incendio del despacho de Eugenio Barruel, por ejemplo, los bomberos tan solo anotaron lo siguiente:

Esta mañana a las 3 1/2 se dio otra vez la alarma de incendio. Esta vez el fuego se había declarado en el despacho N 169 Calle de Yungay. La Bomba salió al mando del Capitán y llegó hasta el lugar amagado de donde regresó al cuartel por haberse estinguido el fuego. Se pasó lista y asistieron 32 Voluntarios, 20 Auxiliares. 52 total. (Tercera Compañía de Bomberos de Valparaíso, 1873)

Aunque este documento era poco útil para esclarecer las causas del incendio y no era incorporado en el sumario, sí revelaba la presencia de 52 personas del cuerpo de bomberos en el incendio. Sin embargo, ninguno de ellos fue requerido como testigo en el tribunal. Los bomberos, hasta entonces, no eran considerados expertos por los tribunales en este tipo de causas. Y, de hecho, casi nunca eran convocados a los estrados judiciales para ofrecer su testimonio.

En cambio, la valoración económica de las pérdidas sí era realizada de manera sistemática por los agentes de las aseguradoras, de hecho, las distintas compañías contaban con manuales de inspección para realizar este tipo de labores. Uno de los que se conserva en lengua castellana es el de la Unión Chilena (Compañía Nacional de Seguros Unión Chilena, 1853). Por ejemplo, en el incendio del despacho de Eugenio Barruel, el agente de seguros Andrés R. Bello procedió inmediatamente, como se indicaba en el manual, a la inspección de la propiedad con el objetivo de comprobar si las perdidas 
coincidían con los elementos declarados en póliza. Este procedimiento era común, y se vería en casos diversos como el de una propiedad pública en la sexta sección de la aduana (Sumario sobre el incendio del 31 de Diciembre y 1 de Enero, 1869), el de la residencia de Estanislao Fraga (Incendio en casa de Don Estanislao Fraga "Población Rocuant", 1898), el de una propiedad comercial, la sombrerería de Maria Elisa Friedman (Incendio en la sombrerería de doña Maria Elisa Friedmann, 1898), e incluso en casos donde no operaba seguro alguno, como el incendio del matadero, se acudía al agente de seguros para que ofreciera su concepto sobre el posible origen del fuego (Incendio en el matadero, 1897). De alguna manera, las aseguradoras habían logrado insertarse de manera sistemática en la discusión jurídica sobre los incendios en Valparaíso.

De hecho, las circunstancias generales de la ciudad de Valparaíso hacían que el incendio fuera casi una condición omnipresente, pues podría presentarse en múltiples condiciones y formas. Esto, a su vez, hacía evidente que alrededor del incendio se articulaban acciones y circunstancias tan distintas que la muerte podía presentarse de muchas maneras. La discrecionalidad, capacidad individual e incluso la arbitrariedad de los jueces, seguiría siendo parte fundamental de la penalización del delito en la práctica.

Más adelante, en el Congreso Nacional el artículo referente a la pena de muerte fue aprobado sin discusión alguna y después de una ligera modificación realizada por el ejecutivo, el artículo quedaría de la siguiente manera: "Art. 474. El que incendiare edificio, tren de ferrocarril, buque u otro lugar cualquiera, causando la muerte de una o mas personas cuya presencia allí pudo prever, será castigado con presidio mayor en su grado máximo a muerte (...)" (Código Penal, 1875, p. 790). El problema fundamental de la comprobación, sin embargo, no quedaba resuelto, por el contrario, la redacción final agregaba otro elemento que la hacía aún más difícil, pues, no solo tendría que constatarse la autoría del crimen, además se debía demostrar que el individuo tuvo la capacidad de prever la presencia de personas en el lugar incendiado aplicando el criterio que considerara más adecuado. La misma situación se presentaba con el artículo 475, el cual decía lo siguiente: "Cuando ejecutare el incendio en edificios, tren de ferrocarril, buque o lugar habitados o en que actualmente hubiere una o mas personas, siempre que el culpable haya podido prever tal circunstancia" (Código Penal, 1875, p. 790). Hasta cierto punto, esta dificultad se daba por la naturaleza misma del incendio, pues era cierto que se trataba de un delito contra la propiedad, pero al mismo tiempo era un delito contra las personas, e igualmente podía considerarse como delito contra la seguridad pública. 
Ninguna de las herramientas para tomar estas decisiones estaba en el Código Penal. Por su parte, la ley de Apreciación de prueba, aprobada en 1876 y que incluía el delito de incendio. tan solo decía lo siguiente:

En todos los procesos criminales que se siguieren por homicidio, hurto, robo, incendios i accidentes de ferrocarriles, tanto los jueces de primera instancia como los Tribunales superiores apreciarán la prueba con entera libertad i absolverán o condenarán al reo, según creyeren en su conciencia que es inocente o culpable. (Apreciación de la prueba en los procesos que se siguieren por homicidio, hurto, etc., 1876, p. 248)

Por lo tanto, el problema se transfería al terreno, pues la ley no ofrecía ninguna herramienta específica para determinar responsabilidades en este tipo de casos. En algunos procesos, como en el incendio del Matadero en agosto de 1898 (Incendio en el matadero, 1897) quedaba perfectamente claro que a la hora del incendio el inmueble estaba totalmente desocupado, pues no había labores en ejecución, el local estaba cerrado y nada permitía concluir que hubiera personas adentro, entonces, quien resultara responsable podría liberarse sin mayor dificultad de las penas de presidio mayor a muerte. Igualmente, en casos como el incendio de la casa de Susana Cruz Díaz (Incendio en el cerro de la cordillera, Callejón largo N²9-31. Casa de Susana Cruz Díaz, 1897), donde era evidente para todos los testigos que había actividad humana al momento de iniciarse el fuego, si hubiera fallecido alguien a causa del incendio, quien resultara culpable sí estaría sujeto a las penas establecidas en estos artículos. Sin embargo, en ninguno de estos casos fue posible encontrar un responsable. Ahora bien, en múltiples casos, como en el incendio del mercado del Cardonal (Sumario por incendio en la plaza del Cardonal, 1902), podía sospecharse que había personal de vigilancia adentro del inmueble, pero ningún indicio señalaba más allá de una duda razonable si había o no personas adentro al momento de iniciarse el fuego.

Naturalmente, el Código no ofrecía todas las herramientas para resolver este tipo de casos, pero sí establecía conceptos que conducían a la necesidad de definir e implementar procedimientos para recabar pruebas. Además, los dos artículos que se vieron anteriormente parecían indicar que los incendios eran delitos contra las personas y su seguridad, y no solamente delitos contra la propiedad, pues además de cuidar la propiedad buscaban proteger la vida de las posibles víctimas. 


\section{PREPARACIÓN Y COMUNICACIÓN DE INCENDIOS}

Según el Código el incendio estaba definido como un delito contra la propiedad. Sin embargo, tanto en la realidad como en el papel, era percibido como algo más. De hecho, el artículo 482 planteaba lo siguiente: "Art. 482. El culpable de incendio o estragos no se eximirá de las penas de los artículos anteriores, aunque para cometer el delito hubiere incendiado o destruido bienes de su pertenencia. (...)" (Código Penal, 1875, p. 791). De esta manera, arruinar la propiedad propia, solo era aceptable siempre y cuando no se hiciera prendiéndole fuego. Este artículo no era difícil de aplicar en la práctica, pero sí señalaba una dificultad referente a la definición misma del concepto de incendio. En efecto, el incendio generaba un resultado que aparentemente superaba su capacidad de dañar la propiedad. Perder, regalar o vender la propiedad propia era un asunto privado, pero incendiarla era un asunto público. Al parecer el acto de prender fuego llamaba la atención de la justicia más allá de la protección de la propiedad ¿Por qué generaba este miedo el incendio? ¿Se temía acaso su carácter incontrolable, su comunicabilidad, o se trataba acaso de un miedo a la dimensión simbólica y política de los grandes incendios? Ciertamente, la experiencia histórica de Valparaíso sentaba un precedente importante en este sentido, pues en 1843, 1850, y 1858 grandes incendios habían destruido importantes sectores de la ciudad. Un incendio pequeño, como el que en 1850 se inició en una cigarrería de la Calle Del $\mathrm{Cabo}^{2}$ y rápidamente se paseó por todo el sector del puerto, demostraba que hasta los más pequeños fuegos descontrolados podían tener consecuencias de grandes proporciones y pérdidas millonarias (Cubillos, 1888, p. 1).

Para esto, además, los comisionados del Código Penal incluyeron los artículos 478 y 479, que referían explícitamente a la comunicación del fuego. Ahora bien, determinar la comunicabilidad desde el punto de vista técnico no ameritaba mayor discusión en la comisión, pero sí se convertiría en un espacio de grandes desacuerdos en los tribunales de Valparaíso. Ejemplo de esto, aunque no se tratara propiamente de un incendio criminal, fue el incendio de las bodegas de Meissener e Hijo en 1906. En este caso, las aseguradoras Alliance Assurance e Imperial Insurance determinaron unilateralmente que el incendio era consecuencia de la comunicación de otros focos causados por el terremoto, ya que se había generado en los días in-

\footnotetext{
${ }^{2}$ Actualmente calle Esmeralda.
} 
mediatamente posteriores al sismo. En su interpretación, la simultaneidad de los hechos era prueba suficiente de la relación de causalidad. Por lo tanto, conforme a la póliza, la indemnización era improcedente. Sin embargo, Vergara Salvá, abogado de Meissener e Hijo, acudió a peritos arquitectónicos para demostrar que los muros cortafuegos y todos los dispositivos legales instalados para impedir la comunicación del fuego estaban conformes a la norma vigente y habían resistido al choque telúrico. De manera que, en su interpretación, aunque ambos eventos hubiesen ocurrido casi sincrónicamente, la relación causa efecto entre el terremoto y el incendio no era demostrable técnicamente (Vergara, 1909). Las aseguradoras, por lo tanto, debían indemnizar a la empresa acorde a la póliza por las pérdidas de mercancía asegurada. La pregunta subyacente a este caso, pero sobre todo a los artículos 478 y 479 del Código, era ¿cómo determinar si un incendiario pudo prever la comunicación de un incendio y cómo determinar culpabilidad en casos de incendios generados en propiedades adyacentes? Es decir, si antes de iniciar el fuego el incendiario constata la existencia de dispositivos cortafuegos, ¿es eso suficiente para liberarlo de las penas por comunicación del incendio?

La pregunta, por el momento, no tenía respuesta, pero sí conducía a pensar en otro elemento que llamaba la atención de los comisionados del Código Penal, la preparación del delito de incendio. En el artículo 481 se planteaba de esta manera:

Art. 481. El que fuere aprehendido con bombas esplosivas o preparativos conocidamente dispuestos para incendiar o causar alguno de los estragos espresados en este párrafo, será castigado con presidio menor en sus grados mínimo a medio; salvo que pudiendo considerarse el hecho como tentativa de un delito determinado debiera castigarse con mayor pena. (Código Penal, 1875, p. 791)

El texto parecía claro, y en la discusión ninguno de los comisionados había visto la dificultad potencial que suponía su aplicación. Efectivamente, en espacios urbanos como Valparaíso era casi imposible determinar cuáles elementos podían considerarse como "preparativos de incendio". Hay que advertir que durante todo el siglo XIX y durante el siglo XX el fuego era una característica central de la vida urbana. Comprar, poseer, transportar y manipular combustibles, fósforos, acelerantes era necesario, prácticamente, en todos los ámbitos de la vida de la ciudad, especialmente en un puerto comercial tan dinámico como Valparaíso. Objetos de uso cotidiano para la 
preparación de alimentos, iluminación, calefacción de espacios, planchado de ropa, manufactura y artesanía, producción industrial y construcción, garantizaban que prácticamente en todos los espacios de la ciudad hubiese, siempre, algún elemento combustible susceptible de explotar o incendiarse. Penalizar preventivamente a un transeúnte por circular con una botella de parafina y una caja de fósforos era imposible en una ciudad como Valparaíso, sin embargo, estos dos elementos eran suficientes para generar grandes incendios.

Para determinar el delito de tentativa de incendio, entonces, era necesario que la disposición de los elementos inflamables fuera explícita y evidente. Por ejemplo, el 20 de noviembre de 1897 en la cantina de Daniels y Halpin en la calle Blanco, la policía constató que la puerta había sido mojada totalmente con parafina, y en el suelo, a un costado, estaban los trapos empapados y la botella utilizada (Tentativa de incendio en la casa calle Blanco 219, cantina de Daniels y Halpin, 1897). Aunque nunca se encontró al responsable, este caso era fácil de resolver, pues la disposición de los elementos inflamables era perfectamente evidente.

Sin embargo, en el caso de la tienda de don Ramón Fábora, que sí se había incendiado, la compañía aseguradora estaba haciendo los trabajos de remoción de escombros para inspeccionar la propiedad de acuerdo con el procedimiento de la empresa, en ese momento encontraron dos trapos que, aparentemente, estaban empapados de parafina y podían ser la causa del incendio. Sin embargo, los colaboradores del agente de seguros procedieron a hacer la comprobación por su propia cuenta quemando los trapos con un fósforo y entorpeciendo el proceso probatorio. La prueba, por lo tanto, no era contundente y estaba alterada, pero tampoco podía descartarse. En ese sentido, el juez ordenó la realización de un análisis de los trapos quemados al químico farmacéutico porteño Emilio Eisele, quien, por su parte, concluía que estos no contenían ni habían contenido aguarrás ni sustancias inflamables derivadas del petróleo (Sumario sobre el incendio de la calle de la matriz esquina con la del arsenal tienda de don Ramón Fabora, 1884). La prueba, entonces, no permitía llegar a conclusiones condenatorias.

Así, mientras que en la cantina de Daniels y Halpin la tentativa de incendio era evidente, en la tienda de Ramón Fábora, el levantamiento de pruebas había sido realizado de manera poco clara por una de las partes interesadas. Por lo tanto, el juez recurría a un análisis experto e imparcial que no resolvía el caso, pero sí permitía descartar la hipótesis de la aseguradora. 


\section{EL INCENDIO CONTABLE}

La dificultad para la comprobación, así como las consecuencias financieras del incendio en propiedades comerciales interesaban especialmente a los comisionados del Código Penal. En ese sentido, redactaron un artículo especial para los incendios en propiedades comerciales aseguradas. El artículo 483 decía lo siguiente: "Se presume responsable de un incendio al comerciante en cuya casa o establecimiento tiene origen aquél, si no justificare con sus libros, documentos u otra clase de prueba, que no reportaba provecho alguno del siniestro." (Código Penal, 1875, p. 791)

El artículo tenía como objetivo invertir la carga de la prueba en casos de incendio relacionados con la cobranza de un seguro en un establecimiento comercial. De esta forma, establecía que aquel comerciante que sufriera un incendio y pudiera recibir una indemnización por ser titular de un seguro debía demostrar que la pérdida era superior a la indemnización. Si bien este artículo, en primera instancia, parecía orientarse a la protección de las compañías aseguradoras, en la práctica, generaba un verdadero desafío contable y hasta ofrecía a los incendiarios fraudulentos mejor preparados una verdadera alternativa para estafar a las compañías. Efectivamente, aquel que de manera premeditada generara una documentación contable fraudulenta y se hiciera cargo de mantenerla a salvo durante el incendio, a diferencia de quien sufriera la quema de sus libros de contabilidad, podría utilizar el artículo 483 a su favor. En la ausencia de pruebas, libros de contabilidad con pérdidas demostrables, aunque fueran falsos, serían mejores evidencias que las cenizas mojadas de una tienda incendiada. Sin embargo, los aseguradores eran conscientes de esto y también estaban preparados para dar la batalla en el plano de la contabilidad.

De hecho, en las propiedades y comercios asegurados, al menos desde 1853, fecha en que se distribuyeron las instrucciones para los agentes de la Unión Chilena, los representantes de cada compañía se mantenían siempre vigilantes. Ya se vio que, en 1868, en el incendio del despacho de Eugenio Barruel, Andrés R. Bello, agente de la Unión Chilena, rápidamente hizo la inspección completa e inventario de la propiedad incendiada. Este documento pretendía constatar que el valor de las mercaderías encontradas era inferior al monto de la póliza. En el juzgado, la inspección y cálculo hechos por Bello sirvieron para que el juez Altamirano condenara a los imputados en primera instancia. Sin embargo, a la fecha no existía la presunción de culpabilidad que establecería posteriormente el Artículo 483, y el abogado David Grimwood lograría la absolución de sus clientes señalándolo de esta manera: 
(...) en nuestro caso no hai disposición alguna legal que establesca la mas lijera presunción contra el que habita una casa que ha tenido la mala suerte de incendiarse, salga o no de ella una y veinte veces si quiere. Y si no hay disposición legal alguna que establesca esta ingrata presunción, no veo porque la dedusia el Ajente Fiscal y sin apoyo legal en que hubiera [de] fundarla. (Grimwood, 1868)

En cambio, después de la promulgación del Código, el procedimiento probatorio cambiaba fundamentalmente. Por ejemplo, para demostrar su inocencia, Berta Tuffy y Elisa Tuffy, imputadas con el cargo de incendio de la sombrerería de su mamá, utilizaron los libros contables de su negocio para demostrar que las pérdidas eran superiores al valor de la póliza suscrita y de esta manera conducían al juez a utilizar el artículo 483 para sobreseer el cargo (Incendio en la sombrerería de doña Maria Elisa Friedmann, 1898). Este tipo de procedimientos contables simples se repetirían prácticamente en todos los casos de incendio en propiedades comerciales desde esta fecha en adelante.

Ahora bien, este artículo se basaba en una práctica habitual en los casos por delito de incendio. Puesto que, si bien los aseguradores ya participaban en gran parte de los procesos, aun cuando las propiedades no estuvieran aseguradas, gracias a dicho artículo, su participación quedaba más claramente definida. En principio, el texto les favorecía, pues la carga de la prueba se invertía y eran los comerciantes quienes debían demostrar su inocencia. En todo incendio en establecimiento comercial asegurado se presumía un comerciante fraudulento. Esto contribuía, ante todo, a que las aseguradoras no tuvieran que incurrir en gastos o procedimientos probatorios difíciles para demostrar intencionalidad en incendios comerciales sin libros de contabilidad.

De esta manera, la discusión penal de incendios se transfería al plano contable generando un cambio fundamental en los procedimientos de demostración. Ejemplo de esto fue el famoso incendio de la tienda "La Americana” del comerciante español Cirilo Íñiguez (Sumario por incendio de la tienda "La Americana" de don Cirilo Iñiguez, 1886), que saldría publicado en los diarios La Unión (“Otro incendio", 1886) y La Patria (Beytia, 1886), que involucraba a una tienda comercial, a una agencia de préstamos, a un propietario inmobiliario y al menos a tres aseguradoras distintas. En este caso, durante varios años los agentes contables de las compañías de seguros y de los negocios incendiados intercambiaron documentación en el juzgado alegando diferencias en los montos de liquidaciones, distintas valoraciones de mercadería, desacuerdos respecto de la calificación de ciertos 
artículos como no-asegurables y diferencias sobre la adición y sustracción de intereses en los montos de las indemnizaciones. Más que la determinación de una responsabilidad penal, la discusión era un verdadero debate de contabilidad y de cálculo matemático.

Otro problema que generaba el artículo 483 se relacionaba con la dificultad para comprobar la existencia de mercaderías en las propiedades incendiadas, pues efectivamente, no era raro que durante los incendios los ladrones aprovecharan para sustraer objetos de los establecimientos comerciales. Así lo había sugerido el abogado de Eugenio Barruel en 1868 (Grimwood, 1868) y por esto mismo se procesaría a José Anjel Herrera en 1884 (Sumario por hurto de mercaderías del incendio de la calle de la Esmeralda, 1884). Sin embargo, en estos casos los informes de las aseguradoras no reportaban robos, pero sí informaban que había mercadería faltante y, de esta manera, al haber un desbalance entre el libro de contabilidad y las mercaderías identificadas en la inspección del incendio, tendían a sugerir intento de fraude.

Este artículo sería discutido años más tarde en la Facultad de Derecho de la Universidad de Chile (Espinoza, 1952; Guzmán, 1938; Naranjo, 1938), y en el Instituto de Ciencias Penales (Valdovinos et al., 1938) resaltándose su inconveniencia práctica. En la Revista de Ciencias Penales, Carlos Valdovinos señalaba que favorecía a comerciantes cautelosos pero poco exitosos en el comercio, que sabían generar libros contables falsos y que lograban contratar pólizas contra incendios más valiosas que sus negocios (Valdovinos, 1938, p. 457). Sin embargo, el artículo no se modificaría sino hasta 1943, y entretanto, todos aquellos comerciantes honestos, asegurados pero desprevenidos que, víctimas del descuido, perdieran sus libros contables junto con los demás enseres en el incendio de su negocio serían presumidos culpables, perderían las indemnizaciones del seguro y deberían pagar las multas y penas correspondientes.

Finalmente, juzgar los incendios parecía ser una tarea tan difícil y diversa como los incendios mismos. Y aunque el Código se presentaba como un instrumento para proteger la vida, la seguridad y la propiedad, la práctica de la justicia en terreno parecía encontrar en él más desafíos y dificultades que herramientas para la demostración de responsabilidades penales.

\section{CONCLUSIONES}

Definir y juzgar el delito de incendio, como se pudo observar a lo largo de este texto, supuso importantes dificultades para la comprobación en Val- 
paraíso. Para empezar, en el artículo, se estableció que una de las motivaciones subyacentes al Código Penal chileno era la protección de inmuebles y mercancías, pues efectivamente, la protección jurídica de la propiedad era un asunto fundamental para la inserción de Valparaíso, y de Chile, en el nuevo comercio global que suponía su ingreso en el capitalismo dependiente (Halperin, 1972, pp. 84-142). Sin embargo, el incendio era un delito complejo, pues podía definirse simultáneamente como un acto y como un resultado. De hecho, un incendio con consecuencias fatales podía resultar de un acto perfectamente cotidiano, incluso sin la intención de hacer daño. Asimismo, en circunstancias incombustibles, como una fría noche de lluvia, un acto deliberado de incendiar podía resultar en una simple tentativa o delito frustrado. El problema jurídico que se desprendía de esta definición era, entonces, ¿ cómo comprobar el delito de incendio consumado si las evidencias de su preparación e ignición eran las primeras en desaparecer? Efectivamente, en la complejidad del delito de incendio pueden observarse las características de vida urbana en sí misma. El incendio y el intento de las instancias judiciales por comprenderlo condujeron a los actores de la época a pensar en las relaciones sociales y en las condiciones geográficas y hasta meteorológicas de las ciudades de Chile.

En el artículo, se pudo constatar que el incendio parecía ser algo más que un delito contra la propiedad. Se observó, por ejemplo, que la comunicabilidad del fuego despertaba en los autores del Código Penal, en los jueces de Valparaíso, en los aseguradores de propiedades y menajes, y hasta en los habitantes de la ciudad, un miedo al incendio que superaba la simple destrucción de la propiedad. Si bien la dimensión cultural del fuego no fue el centro de la argumentación del texto, sí se pudo observar que este elemento que vinculaba tanto lo natural como lo antrópico atañía a miedos profundos producidos por el carácter incontrolable del fuego y por la posibilidad de perderlo todo en un instante. Por lo tanto, el Código establecía artículos pensados para penalizar la comunicación de incendios y la capacidad de prever una conflagración. Pero, más allá del papel, en el juzgado y en el terreno estos textos no parecían ofrecer mayores herramientas a los jueces. ¿Cómo juzgar la capacidad de prever algo tan imprevisible como el comportamiento del fuego? De hecho, incluso con bomberos activos, muros cortafuegos en pie y dispositivos ignífugos en funcionamiento, los incendios de Valparaíso nunca, hasta el día de hoy, han dejado de pasearse de una casa a la siguiente. En ese sentido, el artículo permite abrir nuevas líneas de investigación en las cuales se reflexione en torno a la pregunta por el miedo a lo incontrolable, por categorías que buscan aproximarse a esto 
como el concepto de riesgo, o por las representaciones culturales del fuego en la vida urbana moderna de Valparaíso.

La dificultad para recabar pruebas y para comprobar la participación de individuos en incendios afectaba directamente la protección de la propiedad que buscaba garantizar el Código penal. En Valparaíso, los intereses de las compañías aseguradoras se veían frecuentemente perjudicados por esta situación. En ese sentido, siguiendo las costumbres corporativas desarrolladas en otros países y las directivas emanadas desde las casas matrices de las compañías, las aseguradoras contra incendio establecieron procedimientos de inspección de propiedades orientados a registrar, valorar y contabilizar mercancías para evitar prácticas fraudulentas. Asimismo, implementaron elaboradas estrategias contables para determinar montos de indemnización. Dichas evaluaciones, rápidamente, pasarían a ser parte fundamental del proceso penal. Los agentes de las compañías localizadas en Valparaíso eran requeridos una y otra vez por los jueces criminales, incluso en casos donde no operaba ningún seguro. Así, con el objetivo de resolver aquello que en principio no tenía evidencias, es decir, la responsabilidad penal en un incendio, la justicia chilena acudía a otro tipo de demostraciones más cercanas a la garantía de intereses comerciales de distintos actores económicos de la ciudad, e incluso, de otros países.

Además, el artículo 483 del Código Penal modificaba los objetivos de la demostración en caso de incendio. Al invertir la carga de la prueba, el procedimiento de inspección y comprobación judicial cambiaba completamente y, en la práctica, se transfería al plano contable. De hecho, no era necesario demostrar cómo se había originado el incendio para encontrar un responsable en un establecimiento comercial. En efecto, presumidos culpables, los comerciantes, víctimas de incendio, debían demostrar que no obtenían ganancia alguna de la indemnización correspondiente a la póliza. La comprobación de incendios, de esta manera, hacía confluir una serie de intereses y racionalidades diversas.

Finalmente, aunque el Código pretendía proteger la propiedad y también la vida y la seguridad pública, era tan difícil demostrar la responsabilidad de individuos en este delito que, como lo diría Carlos Valdovinos algunos años después, hacía falta crear una Brigada Técnica especial de incendios en el Laboratorio Criminológico del Servicio de Investigaciones para acudir a lugares incendiados (Valdovinos et al., 1938, p. 463). Adicionalmente, era necesario transferir los esfuerzos de las autoridades de la comprobación a la prevención. Su propuesta buscaba vigilar de cerca el negocio de los seguros, reglamentar e inspeccionar edificios, establecer 
patrullajes permanentes para frustrar incendios, y proporcionar mejores sistemas de alarma para los bomberos (Valdovinos, 1938, p. 461). De esta manera, la historia del delito de incendio que se ha expuesto a lo largo de estas páginas abre un horizonte de investigación que pretende superar la historia del derecho penal. Comprobar la comisión de un delito sin evidencias contundentes implicó, en Valparaíso, sumergirse en los detalles más recónditos de la vida cotidiana de los habitantes de la ciudad a través de los testimonios registrados en los tribunales criminales.

\section{REFERENCIAS}

Aliaga, C., Cerda, J., Foncea, L., Hurtado, S., Lama, A., Órdenes, C., Pizarro, J., Ruiz, J. E., \& Said, J. A. (1974). En A. Bascuñán Valdés (ed.). El delito de incendio. Santiago de Chile: Editorial Jurídica de Chile.

Apreciación de la prueba en los procesos que se siguieren por homicidio, hurto, etc. (1876). Libro XLIV No 1 Boletín de las leyes i decretos $₫$ Tomo 40.

Arango, D. (2019). Valparaíso ignífuga. El urbanismo para la prevención de incendios (1840-1906). Seminario Internacional de Investigación en Urbanismo, 0(11), s.p. Doi: https://doi.org/10.5821/SIIU.6496

Arango, D. (2020, mayo 1). Wooden architecture in a fire prone city. Local Craftsmanship and the Risk of Fire in Valparaíso, Chile 1843-1906. 2020. Society of Architectural Historians Virtual Conference. https://app.oxfordabstracts.com/events/973/program-app/program

Beytia, P. N. (1886, febrero 5). Otro incendio. La Patria.

Brangier, V. (2014). Hacia la construcción de un esquema de administración de justicia en Chile en el Siglo XIX. En M. J. Correa (ed.). Justicia y vida cotidiana en Valparaíso. Siglos XVII-XX (pp. 34-57). Santiago de Chile: Acto editores.

Código Civil (1856). https://www.leychile.cl/Navegar?idNorma=172986\&idPa rte $=8717776 \&$ idVersion $=2000-05-30$

Código Penal (1875). http://www.memoriachilena.gob.cl/602/w3-article-101 18.html

Comisión redactora del código penal. (1873). Actas de la comisión redactora del código penal chileno. Santiago de Chile: Imprenta de la República de Jacinto Núñez.

Compañía Nacional de Seguros Unión Chilena (1853). Instrucciones para los señores ajentes en jeneral. Santiago de Chile: Imprenta de Julio Belin.

Cubillos, M. (1888). Documentos de Cuerpo de Bomberos de Valparaíso. 18501853. Valparaíso: Imprenta americana de Federico T. Lathorp.

Espinoza, A. (1952). Jurisprudencia del delito de incendio 1900-1946 [Memoria de prueba para optar al grado de licenciado]. Universidad de Chile. 
Grimwood, D. (1868). Solicitud de absolución (Caja 20. Expediente 5.). Archivo Nacional de Chile.

Guzmán, A. (1938). El delito de incendio [Memoria de prueba para optar al grado de licenciado]. Universidad de Chile.

Halperin, T. (1972). Hispanoamérica después de la independencia. Consecuencias sociales y económicas de la emancipación. Buenos Aires: Paidós.

Incendio en el matadero, Caja 57. Expediente 4 (Primer juzgado del crimen de Valparaíso 28 de agosto de 1897).

Incendio en el cerro de la cordillera, Callejón largo $\mathrm{N}^{\circ}$ 29-31. Casa de Susana Cruz Díaz, Caja 57. Expediente 7 (Primer juzgado del crimen de Valparaíso 7 de septiembre de 1897).

Incendio en casa de Don Estanislao Fraga "Población Rocuant", Caja 57. Expediente 15 (Primer juzgado del crimen de Valparaíso 1 de enero de 1898).

Incendio en la sombrerería de doña Maria Elisa Friedmann, Caja 57. Expediente 21. (Primer juzgado del crimen de Valparaíso 23 de marzo de 1898).

Intendencia de Valparaíso. (1838). Proyecto para la organización del servicio de las Bombas de Incendio (MINT 181). Archivo Nacional de Chile.

Intendencia de Valparaíso. (1844). Proyecto de reglamento de policía para el Departamento de Valparaíso (MINT 203). Archivo Nacional de Chile.

Llorca, M. (2010). The Marine Insurance Market for British Textile Exports to the River Plate and Chile, c. 1810-50. En R. Pearson. The Development of International Insurance. London: Pickering \& Chato.

Llorca, M. (2011). La historia del seguro en Chile, 1810-2010. Fundación Mapfre.

Naranjo, O. (1938). La presunción del delito de incendio [Memoria de prueba para optar al grado de licenciado]. Universidad de Chile.

Ordenanza contra incendios dictada por la Municipalidad de Valparaíso, Boletín de las leyes y decretos del gobierno $\$$ Libro XXVII. $N^{\circ} 1$ (1859).

Otro incendio. (1886, febrero 5). La Unión, 3.

Sumario sobre el incendio del 31 de Diciembre y 1 de Enero, Caja 20. Expediente 2. (Primer juzgado del crimen de Valparaíso 2 de enero de 1869).

Sumario por hurto de mercaderías del incendio de la calle de la Esmeralda, Caja 111. exp 4 (Segundo juzgado del crimen de Valparaíso 8 de enero de 1884).

Sumario sobre el incendio de la calle de la matriz esquina con la del arsenal tienda de don Ramón Fabora, Caja 102 exp. 09 (Segundo Juzgado del Crimen de Valparaíso 2 de mayo de 1884).

Sumario por incendio de la tienda "La Americana" de don Cirilo Iñiguez, Caja 108. exp. 15 (Segundo juzgado del crimen de Valparaíso 5 de febrero de 1886).

Sumario por incendio en la plaza del Cardonal, Caja 206. Expediente 29 (Segundo Juzgado del Crimen de Valparaíso abril de 1902).

Tentativa de incendio en la casa calle Blanco 219, cantina de Daniels y Halpin, Caja 57. Expediente 3 (Primer juzgado del crimen de Valparaíso 20 de noviembre de 1897). 
Tercera Compañía de Bomberos de Valparaíso. (1873). Libro de Diario 18681873. Archivo de la Tercera Compañía de Bomberos de Valparaíso.

Valdovinos, C. (1938). La prevención de los incendios. Revista de Ciencias Penales 4(23), 455-461.

Valdovinos, C., Scweitzer, M., \& Figueroa, W. (1938). Informe de la Comisión designada por el Instituto de Ciencias penales para estudiar el delito de incendio. Revista de Ciencias Penales 4(23), 461-464.

Vergara, J. de D. (1909). Aseguradores contra asegurados. Dos escritos de un juicio de seguros sobre indemnización por incendio ocurrido en Valparaíso el 17 de Agosto de 1906. Valparaíso: Imprenta del Pacífico. 